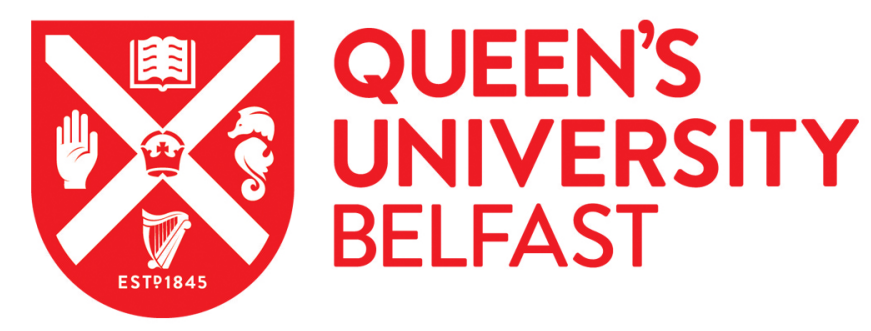

\title{
Solid-liquid interfacial free energy out of equilibrium
}

Cheng, B., Tribello, G. A., \& Ceriotti, M. (2015). Solid-liquid interfacial free energy out of equilibrium. Physical Review B (Condensed Matter), 92(18), 1. [180102]. https://doi.org/10.1103/PhysRevB.92.180102

Published in:

Physical Review B (Condensed Matter)

Document Version:

Publisher's PDF, also known as Version of record

Queen's University Belfast - Research Portal:

Link to publication record in Queen's University Belfast Research Portal

Publisher rights

○2015 American Physical Society

\section{General rights}

Copyright for the publications made accessible via the Queen's University Belfast Research Portal is retained by the author(s) and / or other copyright owners and it is a condition of accessing these publications that users recognise and abide by the legal requirements associated with these rights.

Take down policy

The Research Portal is Queen's institutional repository that provides access to Queen's research output. Every effort has been made to ensure that content in the Research Portal does not infringe any person's rights, or applicable UK laws. If you discover content in the Research Portal that you believe breaches copyright or violates any law, please contact openaccess@qub.ac.uk. 


\title{
Solid-liquid interfacial free energy out of equilibrium
}

\author{
Bingqing Cheng, ${ }^{1}$ Gareth A. Tribello, ${ }^{2}$ and Michele Ceriotti ${ }^{1, *}$ \\ ${ }^{1}$ Laboratory of Computational Science and Modeling, Institute of Materials, École Polytechnique Fédérale de Lausanne, \\ 1015 Lausanne, Switzerland \\ ${ }^{2}$ Atomistic Simulation Centre, School of Mathematics and Physics, Queen's University Belfast, Belfast, BT7 1NN, United Kingdom
}

(Received 8 August 2015; revised manuscript received 30 September 2015; published 9 November 2015)

\begin{abstract}
The properties of the interface between solid and melt are key to solidification and melting, as the interfacial free energy introduces a kinetic barrier to phase transitions. This makes solidification happen below the melting temperature, in out-of-equilibrium conditions at which the interfacial free energy is ill defined. Here we draw a connection between the atomistic description of a diffuse solid-liquid interface and its thermodynamic characterization. This framework resolves the ambiguities in defining the solid-liquid interfacial free energy above and below the melting temperature. In addition, we introduce a simulation protocol that allows solid-liquid interfaces to be reversibly created and destroyed at conditions relevant for experiments. We directly evaluate the value of the interfacial free energy away from the melting point for a simple but realistic atomic potential, and find a more complex temperature dependence than the constant positive slope that has been generally assumed based on phenomenological considerations and that has been used to interpret experiments. This methodology could be easily extended to the study of other phase transitions, from condensation to precipitation. Our analysis can help reconcile the textbook picture of classical nucleation theory with the growing body of atomistic studies and mesoscale models of solidification.
\end{abstract}

DOI: 10.1103/PhysRevB.92.180102

PACS number(s): 64.70.D-, 02.70.Ns, 05.70.Np, 68.08.-p

Solidification underlies many natural phenomena such as the freezing of water in clouds and the formation of igneous rock. It is also crucial for various critical technologies including commercial casting, soldering, and additive manufacturing [1-3]. Computational and experimental studies of these phenomena are complicated by the fact that homogeneous nucleation of solids often occurs at temperatures well below the melting point $T_{\mathrm{m}}$ [4]. The free energy change associated with the formation of a solid nucleus containing $n_{\mathrm{s}}$ particles is usually written as

$$
G\left(n_{\mathrm{s}}\right)=\mu_{\mathrm{s} 1} n_{\mathrm{s}}+\gamma_{\mathrm{sl}} A\left(n_{\mathrm{s}}\right) .
$$

In this expression the first bulk term stems from the difference in chemical potential between the solid and the liquid $\mu_{\mathrm{sl}}=$ $\mu_{\mathrm{s}}-\mu_{1}$, which is negative below $T_{\mathrm{m}}$. The second term describes the penalty associated with the interface between the two phases, and introduces a kinetic barrier to nucleation. This surface term is the product of interfacial free energy $\gamma_{\mathrm{sl}}$, and the extensive surface area $A\left(n_{\mathrm{s}}\right)$. However, due to the diffuse nature of the interface, there is a degree of ambiguity in the location and area of the dividing surface between phases. In classical nucleation theory (CNT), an infinitesimally thin dividing surface divides the solid nucleus from the surrounding liquid. These two phases are usually taken to have their bulk densities so the surface area of the solid nucleus can be calculated using $A\left(n_{\mathrm{s}}\right)=\sigma n_{\mathrm{s}}^{2 / 3}$, where $\sigma$ is a constant that depends on the shape, e.g., $\sigma=(36 \pi)^{1 / 3} v_{\mathrm{s}}^{2 / 3}$ for a spherical nucleus with bulk solid molar volume $v_{\mathrm{s}}$. Under these assumptions, the rate of nucleation can be estimated by calculating the free-energy barrier to nucleation using $G^{\star}=\frac{4}{27} \sigma^{3} \mu_{\mathrm{sl}}^{-2} \gamma_{\mathrm{sl}}^{3}$.

The experimental determination of nucleation rates is challenging due to the nonequilibrium conditions, and is often

\footnotetext{
*michele.ceriotti@epfl.ch
}

affected by considerable uncertainties [4,5]. These difficulties have triggered the development of numerous computational methods for evaluating the free energy change during nucleation. Inducing the formation of a solid nucleus from the melt in an atomistic simulation [6-8] allows one to directly evaluate $G\left(n_{\mathrm{s}}\right)$ at any given temperature, without explicitly partitioning the free energy as in Eq. (1). However, this approach is restricted by the system size, and usually only works at unphysical undercoolings, where the critical nucleus consists of a few hundred atoms. Extrapolating results for $G^{\star}$ obtained from these simulations to the mesoscale critical nuclei formed at experimental conditions inevitably requires invoking models such as CNT, which involves quantities that are ill defined for nanosized nuclei $[9,10]$. In addition, because the nuclei observed in these simulations are tiny, defining their shape and surface area is problematic, which makes it almost impossible to infer the anisotropy of $\gamma_{\mathrm{sl}}$. For these reasons, an alternative strategy in which $\gamma_{\mathrm{sl}}$ is evaluated for an idealized planar interface, which resembles the surface of a mesoscopic nucleus, is often adopted. In the planar limit the value of the surface area $A\left(n_{\mathrm{s}}\right)$ is unequivocal. In addition, different crystallographic orientations can be treated separately, so $\gamma_{\mathrm{sl}}$ and its anisotropy can be defined more rigorously. Techniques of this type include the capillary fluctuation method, which relies on an analysis of the height profile for the atomically rough solid-liquid interfaces $[11,12]$ and the cleaving method, which computes the reversible work required to cleave the bulk solid and liquid and to join the pieces to generate an interface [13-15]. These two methods only allow one to examine how the system behaves at the melting temperature, however.

To investigate the solid-liquid interface at conditions that mirror the ones in experiments, a method that works for planar interfaces and that operates under realistic levels of undercooling is required. In this Rapid Communication, we derive just such a method by extending an approach that uses 
a history-dependent bias to reversibly create and dissolve a solid-liquid interface at equilibrium $[16,17]$ so that it also works at $T \neq T_{\mathrm{m}}$. We then use this method to compute the temperature dependence of $\gamma_{\mathrm{sl}}$ for a realistic interatomic potential.

In order to relate atomic-scale descriptions with mesoscopic models and experiments, it is necessary to introduce a consistent framework for quantifying the number of solid atoms $n_{\mathrm{s}}$. At the atomistic level, several families of structural fingerprints $\phi(i)$ can be used to determine whether the environment around atom $i$ is solidlike or liquidlike [18]. In our simulations, for example, we used a modified version of the cubic harmonic order parameter $\kappa(i)$ introduced in Ref. [16], which, as described in the Supplemental Material [19], we further refined so as to better discriminate between the liquid, the solid, and the various different crystal orientations by applying a switching function $S$ and defining $\phi(i)=S[\kappa(i)]$. Instead of defining $n_{\mathrm{s}}$ by introducing an ad hoc threshold on $\phi$, which is quite common in simulations of this kind, we adopted an alternative approach, which is closely related to the classical definition of a Gibbs dividing surface based on a thermodynamic variable such as the volume $V$. For each microstate, we construct a global collective variable $(\mathrm{CV}) \Phi=\sum_{i} \phi(i)$ by summing over all the atoms. Taking $\phi_{\mathrm{s}}$ and $\phi_{1}$ to be the reference values of the order parameter averaged over atoms in the bulk solid and the bulk liquid at the same thermodynamic conditions, one can then define the number of atoms of solid as

$$
n_{\mathrm{S}}(\Phi)=\left(\Phi-N \phi_{1}\right) /\left(\phi_{\mathrm{s}}-\phi_{\mathrm{l}}\right) .
$$

This is equivalent to choosing a reference state that satisfies $\Phi=\phi_{\mathrm{s}} n_{\mathrm{s}}+\phi_{1} n_{\mathrm{l}}$, subject to the conservation of the total number of atoms in the system $n_{\mathrm{s}}+n_{1}=N$. In other words, zero surface excess for the extensive quantity $\Phi$ has been assumed. Within this approach, the choice of the order parameter implicitly determines the position of the solid-liquid dividing surface while automatically averaging over roughness and thermal fluctuations, which is essential for determining the macroscopic value of $\gamma_{\mathrm{sl}}$ [10].

We simulated the solid-liquid interface for a simple but realistic Lennard-Jones system $[15,16,20]$. To accelerate the sampling so as to obtain reversible formation of a solid-liquid interface in a viable amount of simulation time, we performed well-tempered metadynamics simulations with adaptive Gaussians [21,22], using $\Phi=\sum_{i} S[\kappa(i)]$ (see above) as the CV. A simulation supercell with dimensions commensurate to the equilibrated lattice at the temperature $T$, was employed. This cell was elongated along the $x$ axis which was chosen to be parallel to the crystallographic orientation of the solid-liquid interface. Simulations were run at constant temperature and pressure, with only the $x$ direction left free to fluctuate. Under these conditions, the planar interfaces, whenever present, are always perpendicular to the chosen lattice direction and always have a surface area $A=2 \Delta y \Delta z$ equal to twice the supercell cross section. The sampling has to be restricted to the relevant regions of phase space: the melt, the coexistence state with correctly oriented solid-liquid interfaces, and the defect-free fcc crystal. Choosing an appropriate CV is critical for achieving this end and, in addition, several judiciously tuned restraints are required to prevent the formation of grain boundaries and twin defects. The presence of these defects was already observed in
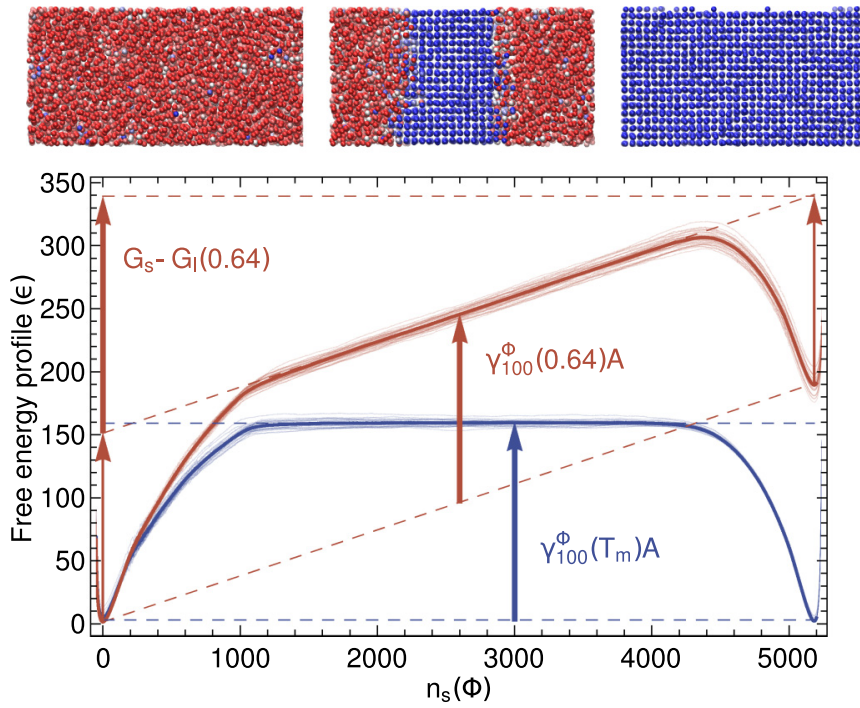

FIG. 1. (Color online) The free-energy profile averaged from 36 independent metadynamics simulation runs, and shown as a function of $n_{\mathrm{s}}(\Phi)$, for an interface perpendicular to $\left.<100\right\rangle$ and a simulation box composed of $16 \times 9 \times 9$ fcc unit cells. Representative atomic configurations for different values of $n_{\mathrm{s}}(\Phi)$ are shown, with atoms colored according to the local order parameter $\phi$. The two sets of curves correspond to the free-energy profiles at the melting temperature $\left[\gamma_{\mathrm{sl}}\left(T_{m}\right)=0.371(2)\right]$ and at a moderate degree of over-heating $\left[\gamma_{\mathrm{sl}}(0.64)=0.365(3)\right]$. All quantities are expressed in Lennard-Jones units.

biased simulations at $T_{\mathrm{m}}$ [17] and they become very likely at undercooled conditions. Fast implementation of this complex simulation setup was made possible by the flexibility of the PLUMED code [23] in combination with LAMMPS [24]. See the Supplemental Material for sample input files and simulation details [19].

Figure 1 shows the free energy $G$ as a function of $n_{\mathrm{s}}(\Phi)$ at two different temperatures. These curves were reconstructed from the metadynamics simulations using on-the-fly reweighting [25,26]. At $T=T_{\mathrm{m}}, G\left[n_{\mathrm{s}}(\Phi)\right]$ displays a broad horizontal plateau that corresponds to the progressive movement of the solid-liquid interface. As discussed in Ref. [16], $\gamma_{\mathrm{sl}}\left(T_{\mathrm{m}}\right) A$ can be unambiguously taken as the difference between the free energy of the plateau and the free energy at the bottom of the solid and liquid minima. The bias potential allows the interface to form even when $\mu_{\mathrm{sl}}$ is nonzero, at temperatures away from $T_{\mathrm{m}}$. Figure 1 thus also reports the free-energy profile computed for $T>T_{\mathrm{m}}$. The free energies of the minima corresponding to the bulk solid and liquid phases now take different values. The slope of the line that joins these minima is equal to $\mu_{\mathrm{sl}}(T)$. The region that was a plateau at $T=T_{\mathrm{m}}$ has become an oblique line, with a slope that is also equal to $\mu_{\mathrm{sl}}$ as moving the dividing surface now has an energetic cost that is associated with forcing atoms to undergo the phase transition [27]. The vertical red arrow in Fig. 1 indicates the free-energy difference between the simulated system with a solid-liquid interface and a reference state composed of $n_{\mathrm{s}}(\Phi)$ atoms of bulk solid and $N-n_{\mathrm{s}}(\Phi)$ atoms of bulk liquid. This quantity is equal to $\gamma_{\mathrm{sl}}(T) A$.

At this point it is important to note that at $T \neq T_{\mathrm{m}}$ a change in the definition of the order parameter $\phi$ affects how 
the free energy of a given state is partitioned between the bulk and surface terms. We will demonstrate later that the value we obtain for $\gamma_{\mathrm{sl}}(T)$ thus depends on $\phi$. As discussed in the Supplemental Material [19], this does not change the free-energy barrier for the formation of a critical nucleus, provided that a consistent framework is used to define the nucleus' size and surface. It can, however, lead to confusion when comparing simulations, phenomenological models, and interpretations of experiments as these may have been analyzed using different definitions. In particular, CNT-based models generally assume that the nucleus has the bulk density. This is equivalent to taking a reference state based on the molar volume, or to using a zero-excess-volume dividing surface. A $V$-based value of $\gamma_{\mathrm{sl}}$ is therefore desirable as one can then establish a direct link between simulations and CNT models. In addition, simulations run with different order parameters can be compared straightforwardly if the volume-based interfacial free energy $\gamma^{V}$ is also computed.

In principle it is possible to compute $\gamma_{\mathrm{sl}}$ using a different local order parameter $\theta$ by on-the-fly reweighing the biased trajectory. In practice, however, any results obtained are only reliable when the sampling of $\Theta=\sum_{i} \theta(i)$ is as thorough as the sampling of the CV used in the metadynamics simulations. In addition, in order to extract $\gamma_{\mathrm{sl}}$, the free-energy profile as a function of $n_{\mathrm{s}}(\Theta)$ must exhibit a clear plateau region that corresponds to solid-liquid coexistence. Unfortunately, the molar volume does not fulfill these two criteria. To illustrate this problem, we performed two-dimensional metadynamics simulations in which $\Phi=\sum_{i} S[\kappa(i)]$ (see above) and the total volume $V$ were used as CVs. In Fig. 2 the resulting free-energy surface is displayed as a function of the solid atom counts obtained when these two extensive quantities are inserted into Eq. (2). The fluctuations in the molar volume for the two bulk phases are so large that they overlap with the plateau region. The molar volume, by itself, is thus not an effective fingerprint for distinguishing the solid and the liquid phases at the atomic scale.

Even though one cannot immediately obtain $\gamma_{\mathrm{sl}}^{V}$ from the free-energy profile as a function of $n_{\mathrm{s}}(V)$, it can still be

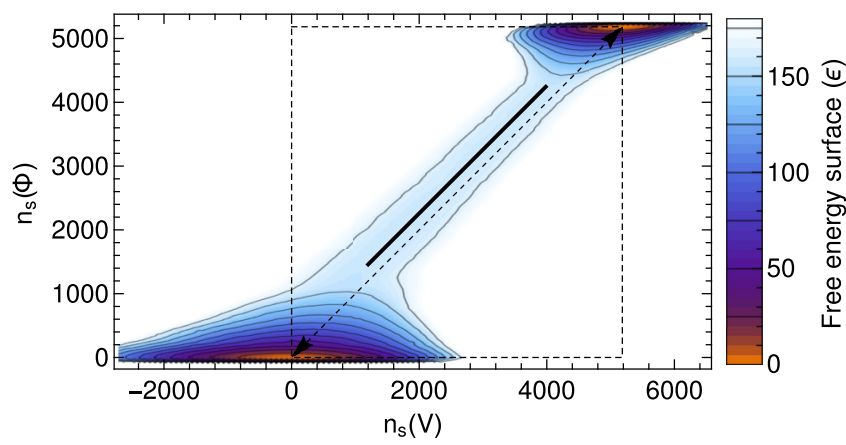

FIG. 2. (Color online) The free energy as a function of $n_{\mathrm{s}}(\Phi)$ and $n_{\mathrm{s}}(V)$ for a simulation at $T_{\mathrm{m}}=0.6185$ computed using a supercell $16 \times 9 \times 9$ times the fcc unit cell. The dashed line indicates the $n_{\mathrm{s}}(\Phi)=n_{\mathrm{s}}(V)$ ideal line, whereas the full line indicates the mean value of $n_{\mathrm{s}}(V)$ for a given value of $n_{\mathrm{s}}(\Phi)$. The horizontal offset between the two curves corresponds to the surface excess for the atom count associated with the $\Phi$-based dividing surface, $\Delta n_{\mathrm{s}}(\Phi, V)$, which for this particular calculation is equal to $259 \pm 2$ atoms. determined indirectly. To do so, one simply computes the change in atom count that occurs when $V$ rather than $\Phi$ is used to define the reference state:

$$
\Delta n_{\mathrm{S}}(\Phi, V)=\left\langle n_{\mathrm{s}}(V) \delta\left[n_{\mathrm{S}}(\Phi)-n_{\mathrm{S}}\left(\Phi^{\prime}\right)\right]\right\rangle-n_{\mathrm{S}}(\Phi) .
$$

In the above, the Dirac $\delta$ ensures that only states with a certain value of $n_{\mathrm{s}}(\Phi)$ are selected, and $\langle\cdot\rangle$ represents a NPT ensemble average, which can be obtained by appropriately reweighting the biased simulation. $\Delta n_{\mathrm{s}}(\Psi, V)$ can be determined to a very high statistical accuracy as long as one of the two order parameters can identify the coexistence region. Graphically, this quantity corresponds to the horizontal offset between the solid black line and the dashed diagonal in Fig. 2, which is constant over the entire plateau region. This implies that, when a solid-liquid interface is present, a change in the definition of the Gibbs dividing surface leads to a constant shift in $n_{\mathrm{s}}$. The interfacial free energy based on the volume, $\gamma_{\mathrm{sl}}^{V}(T)$, can thus be inferred from the quantity $\gamma_{\mathrm{sl}}^{\Phi}(T)$ using $\gamma_{\mathrm{sl}}^{V}=\gamma_{\mathrm{sl}}^{\Phi}-\mu_{\mathrm{sl}} \Delta n_{\mathrm{s}}(\Phi, V) / A$.

Figure 3 shows the temperature dependence for $\mu_{\mathrm{sl}}$ and $\gamma_{\mathrm{sl}}$ computed for the $\langle 100\rangle$ and $\langle 111\rangle$ crystal lattice orientations. $\mu_{\mathrm{sl}}$ is a bulk property so its value should not depend on the orientation of the surface as is observed in the top panel. The magnitude and anisotropy of the interface free energy at $T=$ $T_{\mathrm{m}}$ match the values reported in the literature Refs. [15,16,20]. Its temperature dependence, however, depends strongly on the choice of reference state. The lower panel shows that $\gamma_{\mathrm{sl}}^{\Phi}$ has a near-constant negative slope for both orientations which indicates that the excess entropy associated with the $\Phi$-based dividing surface is positive. The importance of using a consistent reference state when studying the solid-liquid interface in out-of-equilibrium conditions is highlighted by the dramatically different behavior observed when the volume is used to define the reference state. $\gamma_{100}^{V}$ increases with temperature as $\Delta n_{\mathrm{s}}(\Phi, V)$ is large and negative. When the molar volume is used to define the reference state a larger fraction of the interface region is seen as an ordered liquid rather than a disordered solid. The interface for the $\langle 100\rangle$ crystal orientation thus has a negative excess surface entropy. For the $\langle 111\rangle$ orientation, meanwhile, $\Delta n_{\mathrm{s}}(\Phi, V)$ is smaller and the slope of $\gamma_{111}^{V}$ remains negative.

It is important to remember that the total free energy of the nucleus is the only quantity that affects its thermodynamic stability. However, the dependence of $\gamma_{\mathrm{sl}}$ on the dividing surface will have consequences on predictions and data analysis if the same reference state is not used consistently. Usually, CNT models are built assuming that the nucleus' density is constant and equal to its bulk value. To be consistent with this assumption, one should thus use $\gamma_{\mathrm{sl}}^{V}$ when making predictions using this theory. In the Supplemental Material [19] we show that, when using a reference state with a nonzero surface volume excess, additional correction terms must be included on top of the usual CNT expressions. In addition to changing the value of $\gamma_{\mathrm{sl}}$, one must use a density that differs from the bulk value when inferring the surface area of the cluster. This density depends on the nucleus size in a manner that is reminiscent of the well-known Tolman correction, which has been shown to be effective when extrapolating the value of $G^{\star}$ obtained from simulations of nanoscopic nuclei to the mesoscale [10]. 


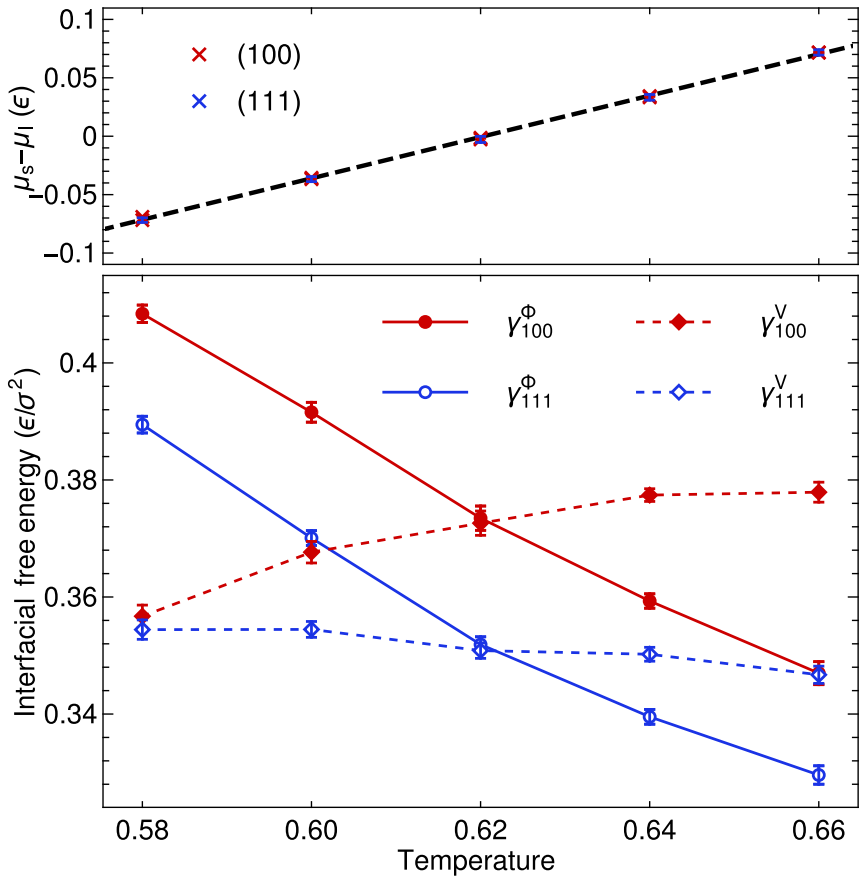

FIG. 3. (Color online) $\mu_{\mathrm{sl}}=\mu_{\mathrm{s}}-\mu_{\mathrm{l}}$ (upper panel) and $\gamma_{\mathrm{sl}}$ (lower panel) for two different crystal orientations, as a function of temperature. $\gamma_{\mathrm{sl}}^{\Phi}$ and $\gamma_{\mathrm{sl}}^{V}$ were computed using a Gibbs dividing surface that has zero surface excess for $\Phi$ and $V$, respectively [see Eq. (2)]. These two different ways of partitioning the free energy of the system between bulk and surface terms ensure that there are significant differences between $\gamma_{\mathrm{sl}}^{\Phi}$ and $\gamma_{\mathrm{sl}}^{V}$ when $T \neq T_{\mathrm{m}}$. Each data point represents an average over 24 independent metadynamics runs and statistical uncertainties are indicated by the error bars. The uncertainties in $\gamma_{\mathrm{sl}}^{\Phi}$ and $\mu_{\mathrm{sl}} \Delta n_{\mathrm{s}}(\Phi, V)$ were assumed to be independent during the error estimation. The data points were obtained by restricting the sampling to prevent complete melting, as discussed in the Supplemental Material [19]. The simulation boxes comprised about 1200 atoms, which implies a small finite-size effect that shifts $T_{\mathrm{m}}$ to 0.62 . At $T_{\mathrm{m}}$ the interfacial free energies for the two orientations we considered are $\gamma_{100}(0.62)=0.373(2)$ and $\gamma_{111}(0.62)=0.352(1)$. All quantities are expressed in Lennard-Jones units.

The observation of a positive slope for $\gamma_{\mathrm{sl}}^{V}(T)$ is consistent with a simple phenomenological model for a hard-sphere system [28], and with the effective $\gamma_{\mathrm{sl}}$ values that were determined by fitting the values of $G^{\star}(T)$ obtained from simulations run at deeply undercooled conditions using a CNT expression [8]. Our results paint a somewhat more nuanced picture, however. The behavior of $\gamma_{100}^{V}$ deviates significantly from linearity even at small undercoolings. In addition, the temperature dependence of the solid-liquid interface energy computed relative to a zero-excess-volume reference depends strongly on orientation, with the $\langle 111\rangle$ direction showing a small negative slope. As a consequence, the anisotropy in $\gamma_{\mathrm{sl}}^{V}$ between the two orientations we considered decreases down to $T=0.58$, suggesting that the shape of the critical nucleus may depend on the degree of undercooling. Fully determining the size and shape of the critical nucleus would require one to determine $\gamma_{\mathrm{sl}}$ and $\partial \gamma_{\mathrm{sl}} / \partial T$ for other high-symmetry orientations and for small misorientations. A direct comparison between planar-interface calculations and three-dimensional nucleation simulations at intermediate undercoolings would also allow one to test the validity of the various assumptions within CNT. For example, such simulations would allow one to determine whether or not the Laplace pressure caused by the curvature of the interface has a significant effect on the chemical potentials of the two phases. These calculations, as well as the investigation of more realistic interatomic potentials, will be the subject of future work, which will provide more accurate parameters for mesoscale phase-field models of nucleation and growth $[29,30]$.

Using a thermodynamic definition for the size of the solid region allows one to treat atomic-scale fingerprints and macroscopic observables on the same footing, thereby providing a practical procedure for converting the value of $\gamma_{\text {sl }}$ obtained with a computationally effective order parameter into the value consistent with the assumptions of mesoscopic theories of nucleation. The combination of this framework and accelerated molecular dynamics makes it possible to generate and stabilize planar interfaces at $T \neq T_{\mathrm{m}}$. Consequently, simulations can be performed with conditions that more closely resemble those in experiments. Such simulations could be used to shed light on the changes in morphology of the critical nucleus, to monitor capillary fluctuations at $T \neq T_{\mathrm{m}}$ [11], and to give atomistic insight on the evolution of interfaces away from equilibrium [31]. In addition, the methodology and the thermodynamic considerations we make here, while examining solidification, would apply with minor modifications to the study of nucleation in other contexts, be it melting [32], precipitation [33], condensation, order-disorder transitions, or other situations in which a phase transition is hindered by a surface energy term [34].

We would like to thank Michel Rappaz for insightful discussion. M.C. acknowledges the Competence Centre for Materials Science and Technology (CCMX) for funding.
[1] J. A. Dantzig and M. Rappaz, Solidification (EPFL Press, Lausanne, 2009).

[2] D. Li, X.-Q. Chen, P. Fu, X. Ma, H. Liu, Y. Chen, Y. Cao, Y. Luan, and Y. Li, Nat. Commun. 5, 5572 (2014).

[3] S. V. Murphy and A. Atala, Nat. Biotechnol. 32, 773 (2014).

[4] D. T. Wu, L. Gránásy, and F. Spaepen, MRS Bull. 29, 945 (2004).
[5] J. J. Hoyt, M. Asta, and A. Karma, Mater. Sci. Eng., R 41, 121 (2003).

[6] P. R. ten Wolde, M. J. Ruiz-Montero, and D. Frenkel, J. Chem. Phys. 104, 9932 (1996).

[7] P. R. ten Wolde and D. Frenkel, Phys. Chem. Chem. Phys. 1, 2191 (1999).

[8] F. Trudu, D. Donadio, and M. Parrinello, Phys. Rev. Lett. 97, 105701 (2006). 
[9] W. Lechner, C. Dellago, and P. G. Bolhuis, Phys. Rev. Lett. 106, 085701 (2011).

[10] S. Prestipino, A. Laio, and E. Tosatti, Phys. Rev. Lett. 108, 225701 (2012).

[11] J. J. Hoyt, M. Asta, and A. Karma, Phys. Rev. Lett. 86, 5530 (2001).

[12] C. A. Becker, D. L. Olmsted, M. Asta, J. J. Hoyt, and S. M. Foiles, Phys. Rev. B 79, 054109 (2009).

[13] J. Q. Broughton and G. H. Gilmer, J. Chem. Phys. 84, 5759 (1986).

[14] R. L. Davidchack and B. B. Laird, Phys. Rev. Lett. 85, 4751 (2000).

[15] R. L. Davidchack and B. B. Laird, J. Chem. Phys. 118, 7651 (2003).

[16] S. Angioletti-Uberti, M. Ceriotti, P. D. Lee, and M. W. Finnis, Phys. Rev. B 81, 125416 (2010).

[17] S. Angioletti-Uberti, J. Phys.: Condens. Matter 23, 435008 (2011).

[18] P. J. Steinhardt, D. R. Nelson, and M. Ronchetti, Phys. Rev. B 28, 784 (1983).

[19] See Supplemental material at http://link.aps.org/supplemental/ 10.1103/PhysRevB.92.180102 for a detailed discussion on how different definition of the dividing surface affects the value of the interfacial free energy as well as the surface area for the 3-D nucleus, a description of the local order parameter used in the biased simulations of our study, and sample input files.

[20] R. Benjamin and J. Horbach, J. Chem. Phys. 141, 044715 (2014).

[21] A. Barducci, G. Bussi, and M. Parrinello, Phys. Rev. Lett. 100, 020603 (2008).

[22] D. Branduardi, G. Bussi, and M. Parrinello, J. Chem. Theory Comput. 8, 2247 (2012).

[23] G. A. Tribello, M. Bonomi, D. Branduardi, C. Camilloni, and G. Bussi, Comput. Phys. Commun. 185, 604 (2014).

[24] S. Plimpton, J. Comput. Phys. 117, 1 (1995).

[25] G. M. Torrie and J. P. Valleau, J. Comput. Phys. 23, 187 (1977).

[26] P. Tiwary and M. Parrinello, J. Phys. Chem. B 119, 736 (2014).

[27] U. R. Pedersen, F. Hummel, G. Kresse, G. Kahl, and C. Dellago, Phys. Rev. B 88, 094101 (2013).

[28] F. Spaepen, Solid State Phys. 47, 1 (1994).

[29] W. A. Curtin, Phys. Rev. Lett. 59, 1228 (1987).

[30] L.-Q. Chen, Annu. Rev. Mater. Res. 32, 113 (2002).

[31] M. E. Gurtin and P. W. Voorhees, Acta Mater. 44, 235 (1996).

[32] A. Samanta, M. E. Tuckerman, T.-Q. Yu, and W. E, Science 346, 729 (2014).

[33] M. Salvalaglio, T. Vetter, F. Giberti, M. Mazzotti, and M. Parrinello, J. Chem. Am. Soc. 134, 17221 (2012).

[34] R. Z. Khaliullin, H. Eshet, T. D. Kühne, J. Behler, and M. Parrinello, Nat. Mater. 10, 693 (2011). 